\title{
Resistance maps for a submicron Hall electrosensor in the diffusive regime
}

\author{
G. Papp ${ }^{\text {a) }}$ \\ Department of Theoretical Physics, University of Szeged, Tisza Lajos körút 84-86, H-6720 Szeged, \\ Hungary; Institute of Physics, University of West Hungary, Bajcsy Zsilinszky út 4-6, H-9400 Sopron, \\ Hungary; and Departement Fysica, Universiteit Antwerpen (CGB), Groenenborgerlaan 171, \\ B-2020 Antwerpen, Belgium \\ F. M. Peeters \\ Departement Fysica, Universiteit Antwerpen (CGB), Groenenborgerlaan 171, B-2020 Antwerpen, Belgium
}

(Received 20 February 2007; accepted 24 April 2007; published online 15 June 2007)

\begin{abstract}
The response of a mesoscopic Hall cross to a local potential barrier is calculated from which we derive two-dimensional resistance maps. At zero magnetic field a double butterfly Hall resistance pattern arises in the Hall cross that is attributed to barrier-induced inhomogeneous current flow. Small, i.e., nonquantizing magnetic fields smoothen out the two wings of the butterfly into a single elongated feature. The longitudinal resistance pattern shows the strongest changes in the current leads leading to two well separated (by the cross area) bumps. Symmetry properties are found for the resistance maps (Hall and longitudinal) with respect to the position of the local introduced potential barrier. (C) 2007 American Institute of Physics. [DOI: 10.1063/1.2745345]
\end{abstract}

\section{INTRODUCTION}

Since its discovery, ${ }^{1}$ the Hall effect has been used to obtain information on the sign and density of charge carriers in, e.g., semiconductors. Conversely, if the transport properties of the semiconductor are well characterized, the Hall effect was recently used to probe the spatial variation of the magnetic field above micromagnets and the flux lines of a superconductor. $^{2-4}$ Similarly when a micromagnet or a mesoscopic superconductor is positioned in the center of such a Hall cross, one obtained information on the response of such objects on, e.g., an external applied magnetic field. ${ }^{5}$ The characterization of a Hall cross as a local detector of magnetic fields was extensively investigated in previous works. $^{6-8}$

Less well known is the use of a Hall cross as a local sensor for electric fields (or potential profiles). A local variation of the potential in the Hall cross results in a local variation of the electron density and consequently of the Fermi velocity. This effect was responsible for the variation of the Hall resistance as first calculated by Baelus et al. ${ }^{9}$ Subsequently, in several experiments an atomic force microscope (AFM) tip was placed above a Hall probe and modified the Hall and bend resistances of submicron Hall cross. ${ }^{10}$ From the response of the Hall cross, information can be obtained on the current flow in such a Hall cross, which is strongly spatial dependent on the presence of a large magnetic field due to the appearance of skipping orbits, or edge states. For small magnetic fields such Hall crosses can also be used as local electric field probes, where recently the important milestone of single electron resolution was achieved at room temperature. $^{11}$

In a recent work, ${ }^{12}$ we calculated the dependence of the Hall and magnetoresistances on the position of a local potential profile from which we obtained two-dimensional (2D)

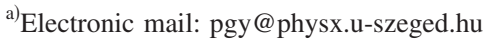

resistance maps which agreed very well with recent experimental measurements. ${ }^{10}$ This calculation was done for a Hall cross in the ballistic regime, i.e., high mobility twodimensional electron gas (2DEG) at low temperature such that $\ell_{e}>2 W\left(\ell_{e}\right.$ is the mean free path of the electrons and $2 W$ is the width of the Hall cross). From previous work, ${ }^{13}$ we know that the effective area of the Hall cross that determines the Hall resistance is twice as large in the diffusive regime as compared to the ballistic regime. This suggests that the response of a submicron Hall cross may be different at room temperature from its known response at cryogenic temperatures. This motivated us to extend our previous work valid in the ballistic regime to the diffusive regime.

The aim of the present paper is to generalize previous studies $^{9,12}$ to the case of diffusive transport (e.g., this is the regime at room temperature) and to calculate the Hall resistance and longitudinal resistance of a Hall cross as function of the position of a local potential profile in the cross junction which is, e.g., induced by a scanning tunneling microscope tip. As a result, we obtain 2D contour plots for the Hall and magnetoresistances which will give us information on the local sensitivity of a Hall cross. Special attention will be paid to symmetry properties of such 2D resistance maps.

This paper is organized as follows. In Sec. II we describe the numerical approach used to determine the Hall resistance and longitudinal resistance of a Hall cross in the diffusive regime in the presence of an arbitrary potential profile placed in an arbitrary position in the Hall cross. In Sec. III the results for zero and in Sec. IV for nonzero magnetic fields are presented for a symmetric Hall cross. Our conclusions are presented in Sec. V.

\section{NUMERICAL APPROACH}

Following Ref. 7 we describe the electrical transport in a two-dimensional electron gas by starting from the steady state continuity equation for the current density $\mathbf{j}$, 


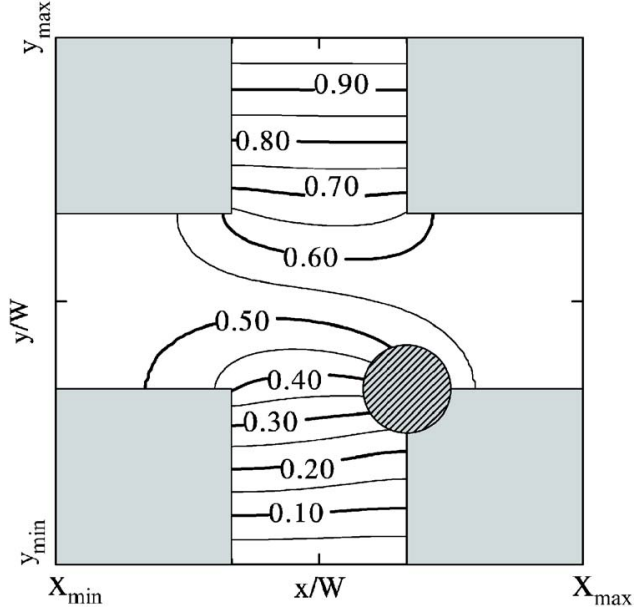

FIG. 1. (Color online) Potential profile arising due to the presence of a Gaussian potential barrier (indicated by the shaded circle) with height $V_{0}$ $=0.8 E_{F}$ and half-width $d=0.25 \mathrm{~W}$. The values on the contour plot are in units of $U$, the applied voltage along the $y$ direction.

$$
\boldsymbol{\nabla j}(x, y)=0,
$$

and use the concept of a local conductivity tensor $\overleftrightarrow{\sigma}(x, y)$ in Ohm's law $\mathbf{j}=\overleftrightarrow{\sigma} \mathbf{E}$. In the presence of a magnetic field normal to the heterojunction plane of the 2DEG, the conductivity becomes a two-dimensional tensor with magnetic field dependent components. Moreover, in the steady state we have $\boldsymbol{\nabla} \times \mathbf{E}=0$ and consequently we can write $\mathbf{E}=-\boldsymbol{\nabla} \Phi$, where $\Phi$ is the electrostatic potential. These expressions substituted in Eq. (1) lead to the following differential equation for the steady state electrostatic potential with a spatially dependent conductivity tensor:

$$
\boldsymbol{\nabla}[\stackrel{\leftrightarrow}{\sigma}(x, y) \boldsymbol{\nabla} \Phi(x, y)]=0 .
$$

The equation can be written in expanded form:

$$
\frac{\partial}{\partial x}\left(\sigma_{x x} \frac{\partial \Phi}{\partial x}+\sigma_{x y} \frac{\partial \Phi}{\partial y}\right)+\frac{\partial}{\partial y}\left(\sigma_{y y} \frac{\partial \Phi}{\partial y}+\sigma_{y x} \frac{\partial \Phi}{\partial x}\right)=0,
$$

with

$$
\overleftrightarrow{\sigma}(\mathbf{r})=\frac{\sigma_{0}(\mathbf{r})}{1+[\mu \mathbf{B}(\mathbf{r})]^{2}}\left[\begin{array}{cc}
1 & \mu B(\mathbf{r}) \\
-\mu B(\mathbf{r}) & 1
\end{array}\right],
$$

where $\sigma_{0}(\mathbf{r})=n(\mathbf{r}) e \mu, n(\mathbf{r})$ is the electron density, and $\mu$ is the mobility. This equation together with appropriate boundary conditions determines the potential $\Phi$. For our Hall cross geometry we demand the following boundary conditions:

$$
\begin{aligned}
& \Phi\left(x, y_{\max }\right)-\Phi\left(x, y_{\min }\right)=U, \\
& j_{x}\left(x_{\min }, y\right)=j_{x}\left(x_{\max }, y\right)=0 .
\end{aligned}
$$

A potential is applied to the Hall device (see Fig. 1) in the $y$ direction and no current can flow through the sides of the sample other than the current contact. After solving Eq. (3) we obtain the potential $\Phi(x, y)$ from which we find the current density $\mathbf{j}(x, y)=-\overleftrightarrow{\sigma}(x, y) \boldsymbol{\nabla} \Phi(x, y)$. Then the current $I$ and the Hall voltage $U_{H}$ can be obtained,

$$
I=\int j_{y}(x, y) d x,
$$

TABLE I. Characteristics of the 2DEG used in two different Hall crosses.

\begin{tabular}{ccccccc}
\hline \hline Sample & $\begin{array}{c}n_{0} \\
\left(\mathrm{~m}^{-2}\right)\end{array}$ & $\begin{array}{c}\mu \\
\left(\mathrm{m}^{2} \mathrm{~V}^{-1} \mathrm{~s}^{-1}\right)\end{array}$ & $\begin{array}{c}\ell_{e} \\
(\mu \mathrm{m})\end{array}$ & $\begin{array}{c}E_{F} \\
(\mathrm{meV})\end{array}$ & $\begin{array}{c}\rho_{0} \\
(\Omega \mathrm{m})\end{array}$ & $\begin{array}{c}R_{c} \\
(50 \mathrm{mT}) / W\end{array}$ \\
\hline $\mathrm{A}$ & $3.3 \times 10^{15}$ & 10 & 1 & 11.79 & 189.4 & 3.8 \\
$\mathrm{~B}$ & $5 \times 10^{15}$ & 9 & 1 & 17.86 & 138.9 & 4.67 \\
\hline \hline
\end{tabular}

$$
U_{H}=\Phi\left(x_{\min }, y\right)-\Phi\left(x_{\max }, y\right),
$$

from which one can calculate the longitudinal resistance $R_{L}$ $=U / I$ and the Hall resistance $R_{H}=U_{H} / I$.

In the presence of a local potential profile, the local electron density in the 2DEG becomes spatial dependent,

$$
n(x, y)=\frac{m^{*}}{\pi \hbar^{2}}\left[E_{F}-V(x, y)\right]=n_{0}\left[1-V(x, y) / E_{F}\right],
$$

where the electron density $n_{0}=\left(m^{*} / \pi \hbar^{2}\right) E_{F}$ of the uniform 2DEG was introduced and $V(x, y)$ is for simplicity taken as a Gaussian potential barrier:

$$
V(\mathbf{r})=V_{0} \exp \left[-\left(\mathbf{r}-\mathbf{r}_{0}\right)^{2} / d^{2}\right],
$$

with height $V_{0}$, width $2 d$ at half maximum, and $\mathbf{r}_{0}$ the center position of the potential barrier in the Hall cross. This results in a region of reduced electron density under the tip and thus a reduced local conductivity accordingly. Using the finite difference techniques, Eq. (3) is solved numerically in the presence of a homogeneous magnetic field with boundary conditions given by Eq. (5).

\section{SYMMETRIC HALL CROSS AT ZERO MAGNETIC FIELD}

The system we envisage is given schematically in Fig. 1: a Hall cross with four identical leads. We consider as an example a Gaussian shape for the local inhomogeneous electric field that will allow us to draw some general conclusions about the electrical response of the Hall cross to a local potential profile as is, e.g., induced by a scanning atomic force microscope tip.

In a typical scanning gate experiment on a Hall cross, the longitudinal and the Hall resistances are measured through macroscopic Ohmic contacts. The conductive tip of an AFM is scanned across the surface with a dc voltage applied with respect to the sample, thereby acting as a local gate that couples capacitively to the sample. The tip-induced potential changes the local potential seen by the conduction band electrons at a position defined by the tip. The resistive responses of the Hall cross are recorded as a function of tip position, which results in so-called scanning gate images. ${ }^{9,10}$ Here, the current and voltage response of the Hall cross will be calculated for the Gaussian potential placed in different grid points at the Hall cross, from which we obtain 2D resistance maps for the Hall $\left[R_{H}\left(\mathbf{r}_{0}, B, V_{0}, d\right)\right]$ and the longitudinal $\left[R_{L}\left(\mathbf{r}_{0}, B, V_{0}, d\right)\right]$ resistances. The calculations were performed for two different Hall crosses and the parameters for the 2DEG are given in Table I, where $n_{0}$ is the electron sheet density, $\mu$ is the electron mobility, $\ell_{e}$ is the mean free 

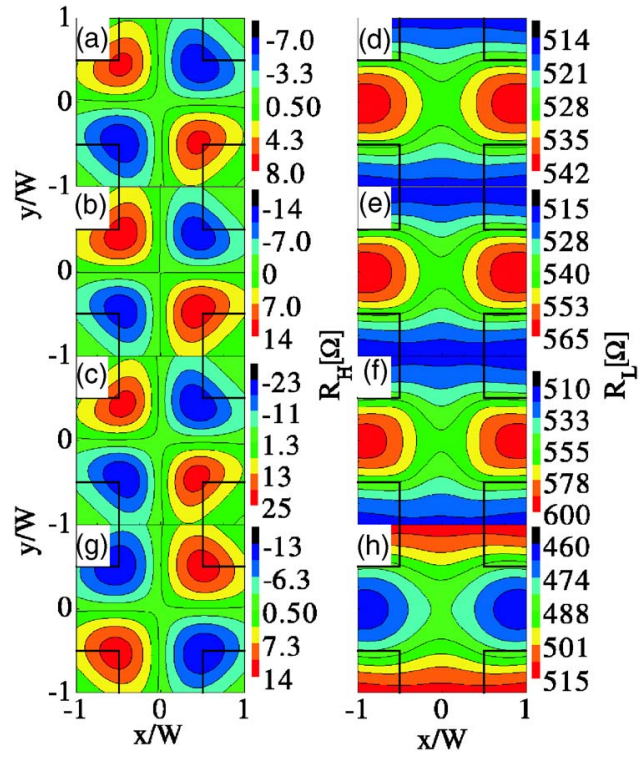

FIG. 2. (Color online) Hall $R_{H}\left(\mathbf{r}_{0}, 0, V_{0}, 0.25\right)$ (left panels) and longitudinal $R_{L}\left(\mathbf{r}_{0}, 0, V_{0}, 0.25\right)$ (right panels) resistance maps for $V_{0}=0.3 E_{F}$ [(a) and (d)]; $V_{0}=0.5 E_{F}\left[(\mathrm{~b})\right.$ and (e)]; $V_{0}=0.7 E_{F}[(\mathrm{c})$ and (f) $] ; V_{0}=-E_{F}[(\mathrm{~g})$ and (h) $]$.

path, $E_{F}$ is the Fermi energy, $\rho_{0}$ is the resistivity, and $R_{c}$ is the cyclotron radius. These quantities correspond to samples used in the measurement of Ref. 10.

In Fig. 2, 2D resistance maps calculated at zero magnetic field are presented for different potential barrier heights $V_{0}$ with constant half-width $d=0.25 \mathrm{~W}$. The corresponding sample parameters are for sample A given in Table I. The left (right) panels show the Hall (longitudinal) resistance maps. The maps show that the Hall resistance, which is zero in a homogeneous sample at $B=0$, can be made nonzero by introducing an inhomogeneity in the sample. The Hall resistance is influenced only near the respective Hall cross area. A very distinct double butterfly (or camel back) type pattern is found which agrees very well with those recently observed in two independent experiments (see Refs. 10 and 11). Qualitatively they are similar to those obtained in the ballistic regime. ${ }^{12}$ Around opposite corners the potential barrier leads to positive and negative Hall resistances with the following symmetries:

$$
\begin{aligned}
R_{H}\left(x_{0}, y_{0} ; 0 ; V_{0} ; d\right) & =R_{H}\left(-x_{0},-y_{0} ; 0 ; V_{0} ; d\right) \\
& =R_{H}\left(-y_{0},-x_{0} ; 0 ; V_{0} ; d\right) .
\end{aligned}
$$

The induced Hall resistance changes depend on the barrier height and, e.g., for $V_{0}=0.5 E_{F}$ are about $\pm 14 \Omega$. For negative barrier height, the positive and negative corners are interchanged but

$$
R_{H}\left(x_{0}, y_{0} ; 0 ; V_{0} ; 0.25\right) \neq R_{H}\left(y_{0}, x_{0} ; 0 ;-V_{0} ; 0.25\right) .
$$

The resistance changes, e.g., for $V_{0}=-E_{F}$ are about $\pm 14 \Omega$. The 2D maps for the longitudinal resistances are depicted in Figs. 2(d)-2(f) with positive and in 2(h) with negative barrier height, respectively. In contrast to the Hall resistance the longitudinal resistance is mainly influenced when the potential probe is positioned outside the Hall cross area and above the current leads. The maxima (minima) are in the current (voltage) probe and these positions are interchanged for

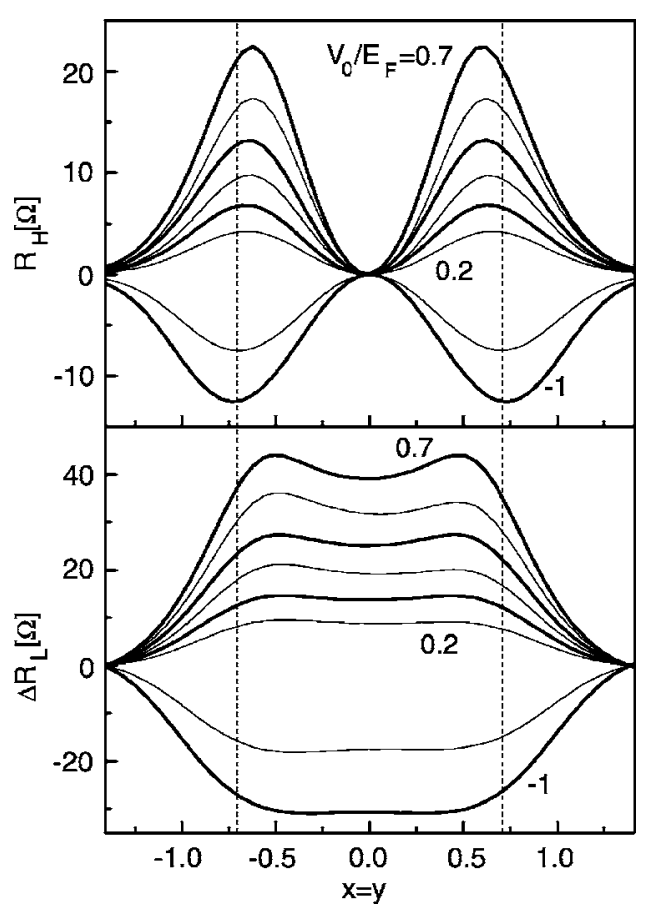

FIG. 3. The $R_{H}$ (top panel) and $\Delta R_{L}$ (bottom panel) as a function of barrier position along the Hall cross diagonal. The vertical dashed curves are the edges of the Hall cross. The different curves are for increasing $V_{0} / E_{F}$ with steps of $0.1(0.5)$ for positive and (negative) $V_{0}$.

negative $V_{0}$. The reason is that a positive voltage barrier reduces the effective width of the current leads when placed in those leads, and consequently resulting in a larger resistance. Notice that for the homogeneous case with $B=0$ and $V_{0}=0$ we have $R_{L}^{0}=2.72 \rho_{0}$ where $\rho_{0}=\sigma_{0}^{-1}$. For a macroscopic sample we know that $R_{L}^{0}=L / W \rho_{0}$, with $L$ the length and $W$ the width of the 2DEG. Here, $L=3 W$ and consequently one would expect $R_{L}^{0}=3 \rho_{0}$. The difference with the numerical result $R_{L}^{0}=2.72 \rho_{0}$ can be understood as follows. The presence of the voltage probes makes the $2 \mathrm{DEG}$ channel locally wider and consequently the effective width of the 2DEG is larger than $W$, resulting in a smaller longitudinal resistance than expected from the above scaling relation. The longitudinal resistance changes induced by the local voltage probe (i.e., potential barrier) depend on its barrier height and, e.g., for $V_{0}=0.5 E_{F}$ are about $[-5 \Omega ; 85 \Omega]$, and $[0 \Omega ;-40 \Omega]$ for $V_{0}=-E_{F}$ and are substantially larger than the variations in the Hall resistance. The figures exhibit the following symmetries:

$$
\begin{aligned}
R_{L}\left(x_{0}, y_{0} ; 0 ; V_{0} ; d\right) & =R_{L}\left(-x_{0}, y_{0} ; 0 ; V_{0} ; d\right) \\
& =R_{L}\left(x_{0},-y_{0} ; 0 ; V_{0} ; d\right) \\
& =R_{L}\left(-x_{0},-y_{0} ; 0 ; V_{0} ; d\right) \\
& =R_{L}\left(-y_{0},-x_{0} ; 0 ; V_{0} ; d\right) .
\end{aligned}
$$

In order to investigate how the maxima of the Hall resistance and the longitudinal resistance changes with $V_{0}$, we plot the $R_{H}$ (top panel) and $R_{L}$ (bottom panel) as a function of barrier position along the Hall cross diagonal for different values of $V_{0} / E_{F}$ in Fig. 3 The maxima of the induced resistance changes increase with increasing $V_{0}$. Notice that for $V_{0} / E_{F}$ $>0$ the maxima move slightly toward the center of the cross 

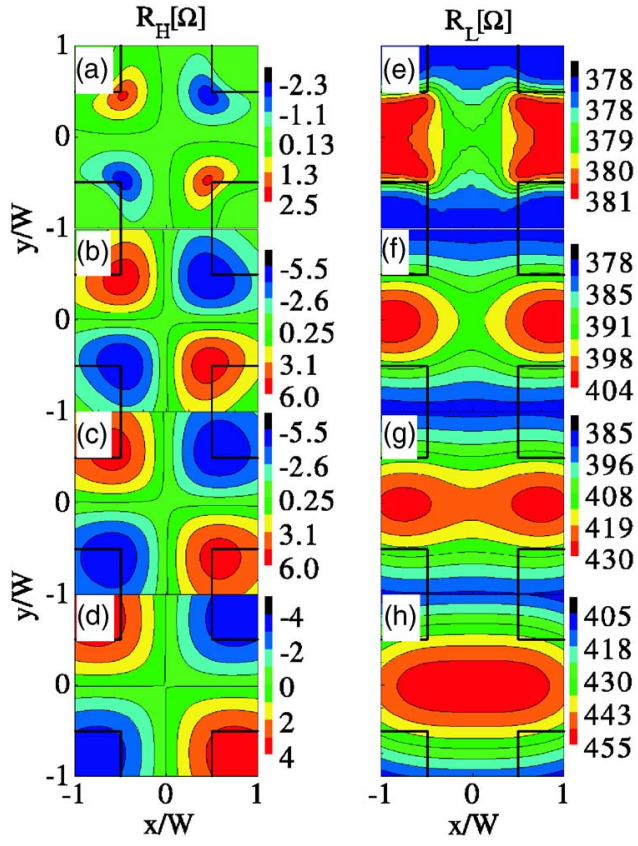

FIG. 4. (Color online) Hall $R_{L}\left(\mathbf{r}_{0}, 0,0.3, d\right)$ (left panels) and longitudinal $R_{H}\left(\mathbf{r}_{0}, 0,0.3, d\right)$ (right panels) resistance maps for $d=0.1 W[(\mathrm{a})$ and (e)]; $d$ $=0.3 W[(\mathrm{~b})$ and (f) $] ; d=0.5 W[(\mathrm{c})$ and $(\mathrm{g})] ; d=0.75 W[(\mathrm{~d})$ and $(\mathrm{h})]$.

with increasing $\left|V_{0}\right|$ while the opposite behavior is found for $V_{0} / E_{F}<0$.

In Fig. 4 we show the 2D maps of Hall [from (a) to (d)] and longitudinal [from (e) to (h)] resistances for different widths $d$ of the potential barrier but fixed potential height $V_{0}=0.3 E_{F}$ at zero magnetic field. The corresponding sample parameters are for sample B given in Table I. Due to the larger zero field conductivity of sample B, i.e., $\sigma_{0}^{B}=n_{0}^{B} e \mu^{B}$ $>\sigma_{0}^{A}=n_{0}^{A} e \mu^{A}$, we found lower resistivities as compared to sample A,

$$
\begin{aligned}
& R_{L}^{A}\left(\mathbf{r}, 0, V_{0}, d\right)>R_{L}^{B}\left(\mathbf{r}, 0, V_{0}, d\right), \\
& R_{H}^{A}\left(\mathbf{r}, 0, V_{0}, d\right)>R_{H}^{B}\left(\mathbf{r}, 0, V_{0}, d\right) .
\end{aligned}
$$

From Fig. 4, e.g., we notice that the longitudinal resistance pattern is very sensitive to the width $d$ of the potential probe: the two detached maxima which are present for $d<0.5 \mathrm{~W}$ merge for larger $d$, e.g., for $d=0.75 W$ [see Fig. 4(h)], into a broad plateau. This feature is illustrated more clearly in Fig. 5(a), where the changes of longitudinal resistances are shown along the $y=0$ axis. In contrast to the longitudinal resistance the Hall resistance, [see Fig. 5(b)] still exhibits its camel back profile with increasing $d$, but the width of the extrema increases with increasing $d$. Also the position of maximaminima is pushed along the diagonals out of the respective Hall cross area with increasing $d$.

\section{SYMMETRIC HALL CROSS FOR NONZERO MAGNETIC FIELD}

Calculations at small, i.e., nonquantizing magnetic fields perpendicular to the 2DEG plane were performed at fixed $V_{0}$ and $d$. 2D maps of Hall resistance at nonzero magnetic fields are presented in Fig. 6. Figures 6(a)-6(f) are for positive field, and Figs. 6(g) and 6(h) are for the cases that the field
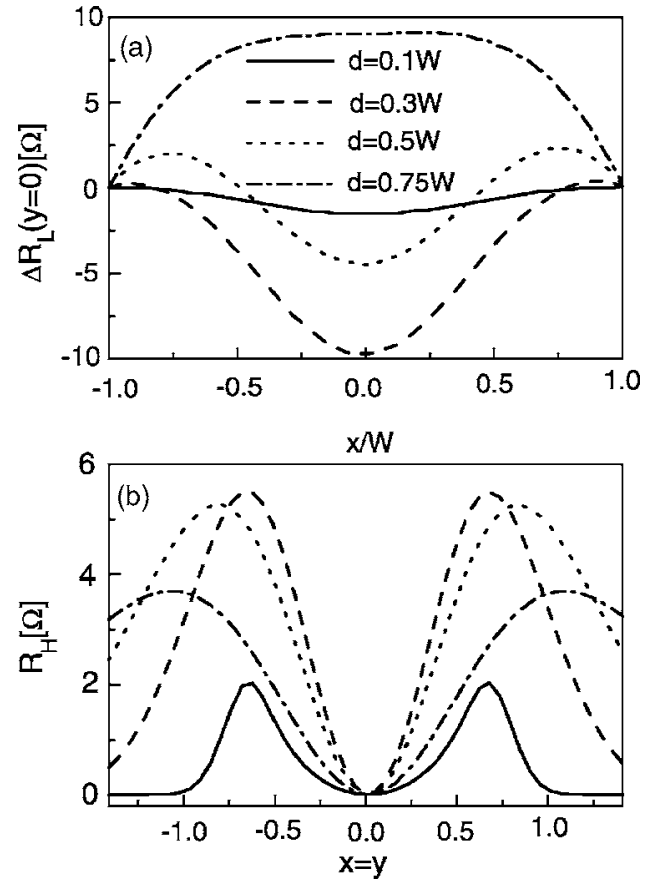

FIG. 5. Longitudinal resistance change as a function of barrier position along the $y=0$ line (a) and the induced Hall resistance change along the diagonal of the Hall cross (b) for fixed $V_{0} / E_{F}=0.3$ and different widths of the Gaussian potential profile.

polarity is reversed. For very small fields the results are qualitatively similar to the $B=0$ case [see Fig. 6(a)] with the difference that, e.g., for $25 \mathrm{mT}$ the induced Hall resistance change is positive. At $B=50 \mathrm{mT}$ [the corresponding cyclotron radius $R_{c}=v_{F} / \omega_{c}=(\hbar / e)\left(k_{F} / B\right)$ with $\left.k_{F}=\sqrt{2 \pi n_{e}}\right]$, the two positive peaks at opposite corners in Fig. 6(a) have started to merge across the center of the Hall cross. The minima at the other corners are still visible in Fig. 6(c). A similar situation is seen for $B=75 \mathrm{mT}$, though the features at the corners are less prominent. Up to this field, the maximum
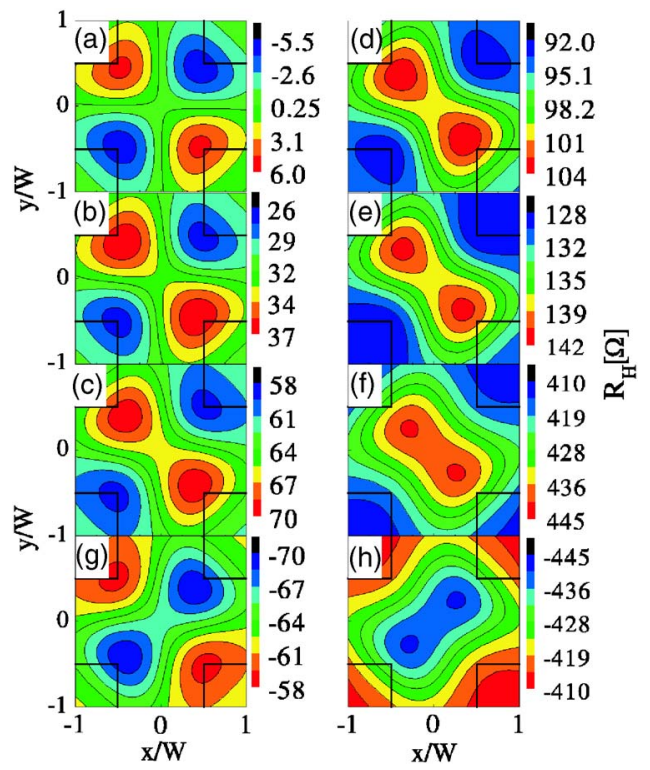

FIG. 6. (Color online) Hall $R_{H}\left(\mathbf{r}_{0}, B, 0.3,0.25\right)$ resistance maps for (a) $B$ $=0 \mathrm{mT}$, (b) $B=25 \mathrm{mT}$, (c) $B=50 \mathrm{mT}$, (d) $B=75 \mathrm{mT}$, (e) $B=100 \mathrm{mT}$, (f) $B$ $=250 \mathrm{mT}$, (g) $B=-50 \mathrm{mT}$, and $(\mathrm{h}) B=-250 \mathrm{mT}$. 


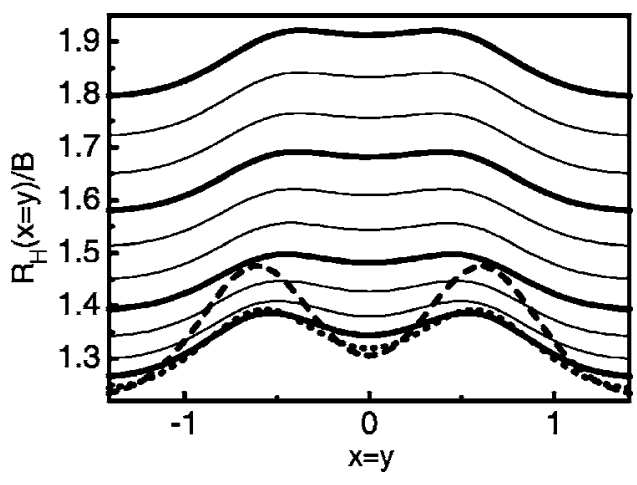

FIG. 7. The scaled Hall resistance $R_{H} / B$ for different values of $B$ vs the barrier position $\left(V_{0} / E_{F}=0.3, d / W=0.25\right)$ along the Hall cross diagonal. The dashed line for $B=25 \mathrm{mT}$, and the dotted line for $B=50 \mathrm{mT}$. The (thick) lines are incremented by steps of $(75 \mathrm{mT}) 25 \mathrm{mT}$ from $(B=75 \mathrm{mT}) B$ $=25 \mathrm{mT}$ to $B=300 \mathrm{mT}$.

variations in the Hall resistance as a consequence of the presence of the potential barrier are always around $12 \Omega$. At $B$ $=100 \mathrm{mT}$ the two maxima are moved closer to the center along the diagonal. Two maxima between the corner and the center are surrounded by global high resistance points at $250 \mathrm{mT}$, and any minima or maxima at the corners have vanished at $B=300 \mathrm{mT}$ [see, e.g., Fig. 10(a)]. In this image one finds a maximum elongated along the diagonal which connects the maxima at $B=0$, with a smooth drop outside the Hall cross area.

We compare the images obtained at positive magnetic fields to those calculated for reversed magnetic fields. A selection of maps is shown in Figs. 6(g) and 6(h) for $B=-50 \mathrm{mT}$ and $B=-250 \mathrm{mT}$. The following symmetry relations are found:

$$
\begin{aligned}
R_{H}\left(x_{0}, y_{0} ; B, V_{0}, d\right) & =-R_{H}\left(-x_{0}, y_{0} ;-B, V_{0}, d\right) \\
& =-R_{H}\left(x_{0},-y_{0} ;-B, V_{0}, d\right), \\
R_{H}\left(x_{0}, y_{0} ; B ; V_{0} ; d\right) & =R_{H}\left(-x_{0},-y_{0} ; B ; V_{0} ; d\right) \\
& =R_{H}\left(-y_{0},-x_{0} ; B ; V_{0} ; d\right),
\end{aligned}
$$

which are identical to those recently found ${ }^{12}$ for the ballistic regime.

In order to see more clearly how the Hall resistance changes with $B$, we plot in Fig. 7 the scaled Hall resistance $R_{H} / B$ for different values of $B$ versus the barrier position along the Hall cross diagonal. We notice the qualitative different behavior for low magnetic field values. It is clearly shown that the zero field image is preserved for $25 \mathrm{mT}$ (see dashed line in Fig. 7) and that the two positive peaks occurring at opposite corners in Fig. 6(a) merge across the center of the Hall cross with increasing magnetic field.

The corresponding 2D maps for the longitudinal resistance at nonzero magnetic fields are presented in Fig. 8 with the same parameters as in Fig. 6. The longitudinal resistance $R_{L}$ is seen to increase with field. Maxima (minima) are found in the voltage (current) probes. Besides the above symmetries, another symmetry can be observed from Figs. 8(c), $8(\mathrm{~g}), 8(\mathrm{f})$, and $8(\mathrm{~h})$ :
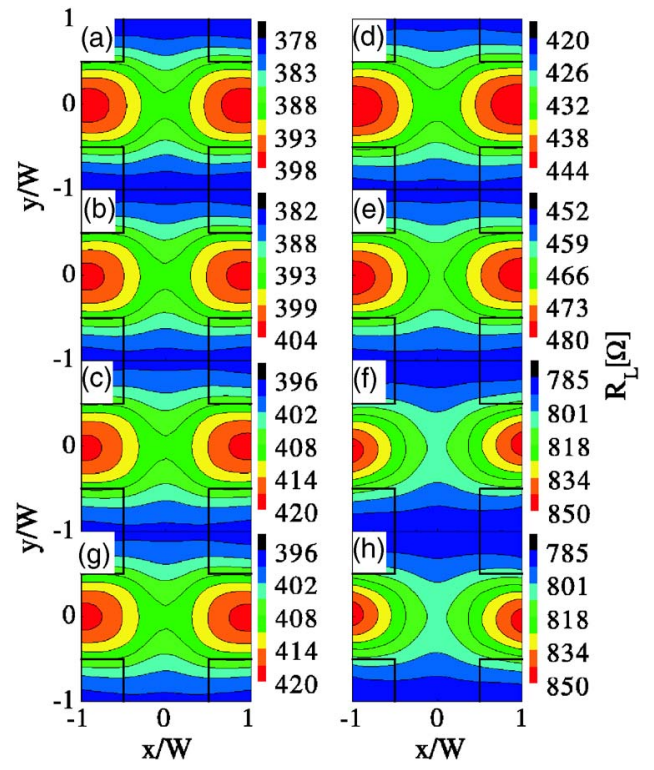

FIG. 8. (Color online) Longitudinal $R_{L}\left(\mathbf{r}_{0}, B, 0.3,0.25\right)$ resistance maps for (a) $B=0 \mathrm{mT}$, (b) $B=25 \mathrm{mT}$, (c) $B=50 \mathrm{mT}$, (d) $B=75 \mathrm{mT}$, (e) $B=100 \mathrm{mT}$, (f) $B=250 \mathrm{mT}$, (g) $B=-50 \mathrm{mT}$, and (h) $B=-250 \mathrm{mT}$.

$$
R_{L}\left(\mathbf{r}_{0}, B, V_{0}, d\right)=R_{L}\left(\mathbf{r}_{0},-B, V_{0}, d\right),
$$

thus the longitudinal resistance is an even function of the magnetic field as expected. Next we investigate the effect of the magnetic field on the longitudinal resistance when the potential barrier is put in the center of the Hall cross (see Fig. 9 ). The effect of the position of the barrier along the middle of the voltage probe is shown by inset (a) of Fig. 9. In the small $B$ region the longitudinal resistance at the center is found to be quadratic in $B$. The behavior of the maxima is similar as can be seen in inset (b) of Fig. 9 .

To investigate the effect of the height of the potential barrier, we performed similar calculations at a fixed magnetic field of $B=300 \mathrm{mT}$ and at fixed half-width $d$ for different barrier heights. Selection of data is presented in Fig. 10. With negative barrier heights (right panels of Fig. 10) the Hall

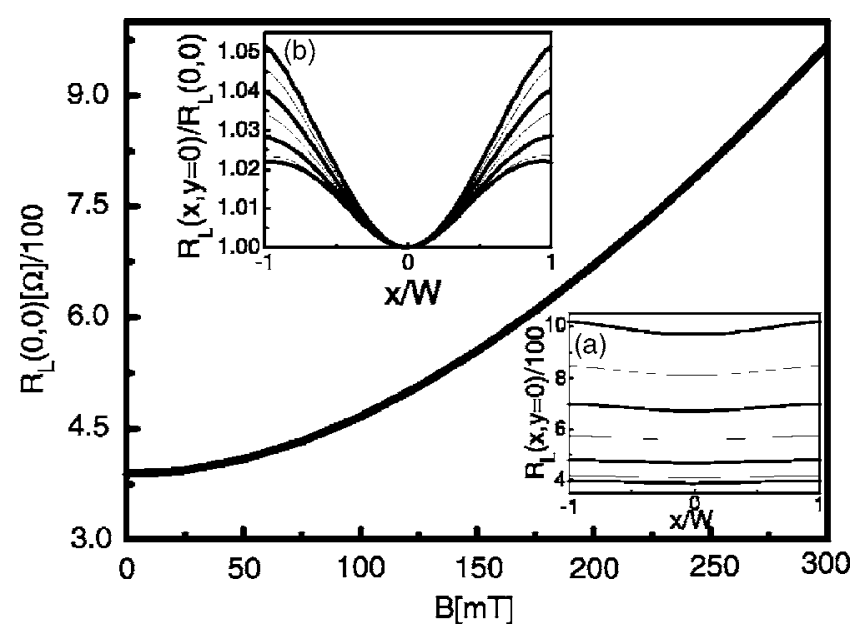

FIG. 9. The longitudinal resistance at the center of the Hall cross vs $B$. The insets show (a) longitudinal resistance as function of barrier position along the $y=0$ line $R_{L}(x, y=0)$ for different $B$. (b) The $R_{L}(x, y=0)$ scaled by $R_{L}(0,0)$. The (thick) lines are incremented by steps of $(100 \mathrm{mT}) 50 \mathrm{mT}$ from $B=0 \mathrm{mT}$ to $B=300 \mathrm{mT}$. 

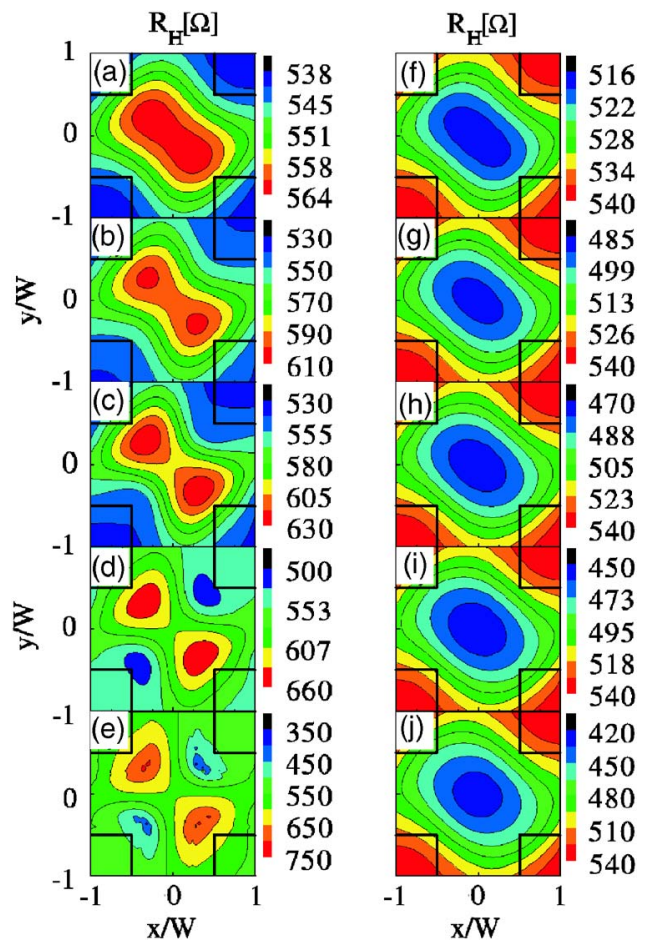

FIG. 10. (Color online) Hall $R_{H}\left(\mathbf{r}_{0}, 300 \mathrm{mT}, V_{0}, 0.25\right)$ resistance maps for (a) $V_{0}=0.2 E_{F}$, (b) $V_{0}=0.5 E_{F}$, (c) $V_{0}=0.7 E_{F}$, (d) $V_{0}=E_{F}$, (e) $V_{0}=1.5 E_{F}$, (f) $V_{0}=-0.2 E_{F}$, (g) $V_{0}=-0.5 E_{F}$, (h) $V_{0}=-0.7 E_{F}$, (i) $V_{0}=-E_{F}$, and (j) $V_{0}$ $=-1.5 E_{F}$.

resistance decreases when the barrier approaches the center of the Hall cross and its minimum value is found when the barrier is in the center of the Hall cross. Notice that the maxima in the four corners are independent of the barrier strength and the minima decrease with increasing $\left|V_{0}\right|$. For positive barrier height it behaves differently (left panels of Fig. 10): with low barrier height the Hall resistance has a global maximum in the center. Increasing the barrier height one can recover those images which correspond to the low magnetic field map, that is, the double butterfly pattern (see Figs. 2 and 6).

\section{CONCLUSION}

We calculated the changes in the longitudinal and Hall resistances of a Hall cross in the diffusive regime in the presence of a local potential barrier which was modeled by a Gaussian function. The effect of the position of such a local potential probe at the Hall cross on the Hall and longitudinal resistances was obtained leading to two-dimensional (2D) resistance maps. The real-space patterns of the induced resistance changes are manifestations of the symmetry properties of such a Hall system. At zero magnetic field a double butterfly Hall resistance pattern arises around the corners of the Hall cross with positive and negative resistances. The size of the induced Hall resistance changes and the positions of the maxima and minima depend on the barrier height. The latter are also influenced by the width of the potential barrier. The largest changes in the longitudinal resistance are found when the center of the potential probe is positioned outside the Hall cross area and toward the current leads. The maxima (minima) are in the current (voltage) probe and are interchanged for negative $V_{0}$. For small magnetic fields (less than $50 \mathrm{mT}$ ) the results are qualitatively similar to the $B=0$ case with the difference that the induced Hall resistance change is positive. The double butterfly structure disappears for higher magnetic field and the two maxima between the corner and the center are surrounded by global high resistance points. But this process can be inverted for a fixed magnetic field value: increasing the barrier height one can recover those images which correspond to the low magnetic field map, but the Hall resistance values are higher. The longitudinal resistance increases with field and in the center of the Hall cross is found to be quadratic in $B$ for small $B$ values.

From the present calculation we found that the Hall cross can be used to locally probe electric fields (or charge). This may also be used to calibrate the potential profile induced by, e.g., an AFM tip. The present results tell us that the Hall cross area has an inhomogeneous sensitivity where regions near the corners of the Hall cross are more sensitive than other regions in the Hall cross. ${ }^{14}$

The obtained resistance maps are those found in the absence of any potential fluctuations which may be inherently present in the 2DEG (e.g., due to fluctuations in the position of the dopants and/or interface fluctuations). Conversely, any experimental found deviations from the theoretical images presented in this paper may learn us about the static inhomogeneities that are present in the 2DEG of the Hall cross.

\section{ACKNOWLEDGMENT}

This work was supported by the Belgian Science Policy (IUAP-program).

${ }^{1}$ E. Hall, Am. J. Math. 2, 287 (1879).

${ }^{2}$ A. Gral, S. J. Bending, and M. Henini, J. Vac. Sci. Technol. B 14, 1202 (1996).

${ }^{3}$ S. J. Bending, Adv. Phys. 48, 449 (1999).

${ }^{4}$ S. Wirth and S. von Molnár, Appl. Phys. Lett. 76, 3283 (2000).

${ }^{5}$ A. K. Geim, S. V. Dubonov, I. V. Grigorieva, J. G. S. Lok, J. C. Maan, X. Q. Li, F. M. Peeters, and Yu. V. Nazarov, Superlattices Microstruct. 23, 151 (1998); A. K. Geim, S. V. Dubonov, I. V. Grigorieva, J. G. S. Lok, J. C. Maan, A. E. Filippov, and F. M. Peeters, Nature (London) 390, 259 (1997).

${ }^{6}$ F. M. Peeters and X. Q. Li, Appl. Phys. Lett. 72, 572 (1998).

${ }^{7}$ I. S. Ibrahim, V. A. Schweigert, and F. M. Peeters, Phys. Rev. B 57, 15416 (1998); S. J. Bending and A. Oral, J. Appl. Phys. 81, 3721 (1997).

${ }^{8}$ P. S. Deo, F. M. Peeters, and V. A. Schweigert, Superlattices Microstruct. 25, 1195 (1998).

${ }^{9}$ B. Baelus and F. M. Peeters, Appl. Phys. Lett. 74, 1600 (1999).

${ }^{10}$ A. Baumgartner, T. Ihn, K. Ensslin, G. Papp, F. M. Peeters, K. Maranowski, and A. C. Gossard, Phys. Rev. B 74, 165426 (2006).

${ }^{11}$ I. I. Barbolina et al., Appl. Phys. Lett. 88, 013901 (2006).

${ }^{12}$ G. Papp and F. M. Peeters, J. Appl. Phys. 101, 063715 (2007).

${ }^{13}$ J. Reijniers and F. M. Peeters, J. Appl. Phys. 87, 8088 (2000).

${ }^{14}$ Y. Cornelissens and F. M. Peeters, J. Appl. Phys. 92, 2006 (2002). 